\title{
Ruptured cerebral mycotic aneurysm, an unusual infective endocarditis presentation
}

\author{
Tiago Alves ${ }^{1}$, Rita Ivo², Marta Rebelo², Eduarda Carmo² \\ ${ }^{1}$ Centro Hospitalar do Médio Tejo. ${ }^{2}$ Centro Hospitalar Lisboa Ocidental
}

\begin{abstract}
Although septic embolization associated with infective endocarditis is relatively frequent, mycotic cerebral aneurysms are a rare and potentially fatal complication. The authors report the case of a woman admitted with a cerebral haemorrhage due to mycotic aneurysm rupture, which led to a subacute infective endocarditis diagnosis. The patient underwent craniotomy with aneurysm excision and mitral valvuloplasty due to severe valvular insufficiency, with a favorable clinical outcome. The authors make a brief review, highlighting the challenge of the management of these patients, especially at surgical approach, which requires an individualized therapy strategy based on patient evolution.
\end{abstract}

Keywords: mycotic, aneurysm, infective endocarditis. Intracranial haemorrhage.

Palabras clave: Aneurisma micótico. Endocarditis infecciosa. Hemorragia intracranial.

\section{INTRODUCTION:}

Septic embolization associated with infective endocarditis (IE) is relatively frequent, however vascular wall involvement by septic emboli is a rare condition, especially at the brain. The term mycotic aneurysm (MA), coined in 1885 by Osler, defines an aneurysmal degeneration of an artery caused by vascular wall inflammation through septic embolization in patients with $\mathrm{E}^{1}$. The mycotic designation results from the "fresh fungus vegetation" appearance and not from the etiological agent'. MA present high morbidity and mortality due to the risk of rupture. The authors describe the case of a patient with subacute IE complicated by ruptured MA.

\section{CASE REPORT}

A 20-year-old woman, previously healthy, admitted at the Emergency Department with a high intensity $(9 / 10)$ holocranial headache, associated with vomiting and drowsiness. The patient also reported episodes of fever, with no defined pattern and partial response to antipyretics, asthenia and weight loss (10\% of initial weight), with 2 months of evolution. In these period, the patient was medicated with antibiotics, whose names she couldn't tell, but without symptomatic improvement.

At the emergency room, the patient was drowsy with a Glasgow Coma Scale: 14points, with isochoric pupils, but presenting a left homonymous hemianopsia at the neurological examination, without others deficits. Febrile $\left(\mathrm{TT}: 38.5^{\circ} \mathrm{C}\right)$, normotensive $(105 / 62 \mathrm{mmHg})$, tachycardic (105bpm) and eupneic. The skin and mucous membranes were pale, without clinical signs of poor peripheral perfusion. At the cardiac exam was recognized an holosystolic murmur, grade III/VI, predominant in the mitral focus and with irradiation to the axilla. The remaining physical exam was normal.

Laboratory results showed leukocytosis (24.500/ul) with neutrophilia (19.900/ul), erythrocyte sedimentation rate: $79 \mathrm{~mm} / \mathrm{h}, \mathrm{C}$ reactive protein:11.9mg/dl and microcytic anemia $(\mathrm{Hb}: 10.2 \mathrm{~g} / \mathrm{d}$, VGM:79.2fl).

Due to the symptomatology and neurological findings a head computerized tomography (CT) scan was performed, revealing an acute intraparenchymal hematoma occipital corticossubcortical and right parietal with extravasation to the ventricular system with a maximum diameter of $5.8 \mathrm{~cm}$ in the axial plane, causing deformation of the supratentorial ventricular system and subfalcine herniation with a midline shift of $5 \mathrm{~mm}$ to the left. There was also a collapse of the quadrigeminal and peri-mesencephalic cisterns, along with ectasia of the ventricular system, evidencing hydrocephalus (figure 1). The angiography revealed an oval distal mycotic aneurysm in the right posterior cerebral artery (parieto-occipital branch) with a $3.5 \mathrm{~mm}$ diameter (figure 2).

The Neurosurgery team was called and the patient underwent a craniotomy with hematoma drainage and aneurysm excision. For the postoperative care the patient was transferred to the Intensive Care Unit and an etiological investigation was performed.

The transthoracic echocardiogram showed a voluminous vegetation, with $21 \mathrm{~mm}$ at the greater axis, adherent to the atrial face of the posterior leaflet of the mitral valve, leading to a severe mitral regurgitation in the Doppler-Corgrated and PISA analysis $\left(E R O A=0.6 \mathrm{~cm}^{2}, V r e g=68 \mathrm{~mL}\right)$ and mild to moderate left atrial enlargement (Volume index $47 \mathrm{~mL} / \mathrm{m}^{2}$ ). Based on the available data, with a probable $\mathbb{E}$ according to the modified Duke criteria. Blood samples were obtained for blood cultures and empirical antibiotic therapy with vancomycin, flucloxacillin and gentamicin was started. The multiples blood cultures remained negative after prolonged incubation, as well as the Coxiella burnetii, Bartonella, Toxoplasma, Legionella, Mycoplasma and Chlamydia spp serologic tests. The autoimmune study, with antinuclear antibodies, antiphospholipid antibody and rheumatoid factor, was also negative. The only positive result found was a high antistreptolysin 0 title $(515 \mathrm{IU} / \mathrm{mL})$ at the admission, which doesn't allow to state a Streptococcus group bacteria aetiology, but may suggest his involvement. Due to severe mitral regurgitation, Cardiothoracic Surgery was contacted and, after a multidisciplinary decision, the surgery was postponed at this stage. The recent cerebral haemorrhage was a contraindication for the anticoagulation required for surgery, which would imply extracorporeal circulation. A CT scan was repeated within 48 hours showing sero-aero-haematic residues from craniotomy, aneurysm excision and no hydrocephalus or midline shift. 
Figure 1. Acute intraparenchymal hematoma occipital corticossubcortical

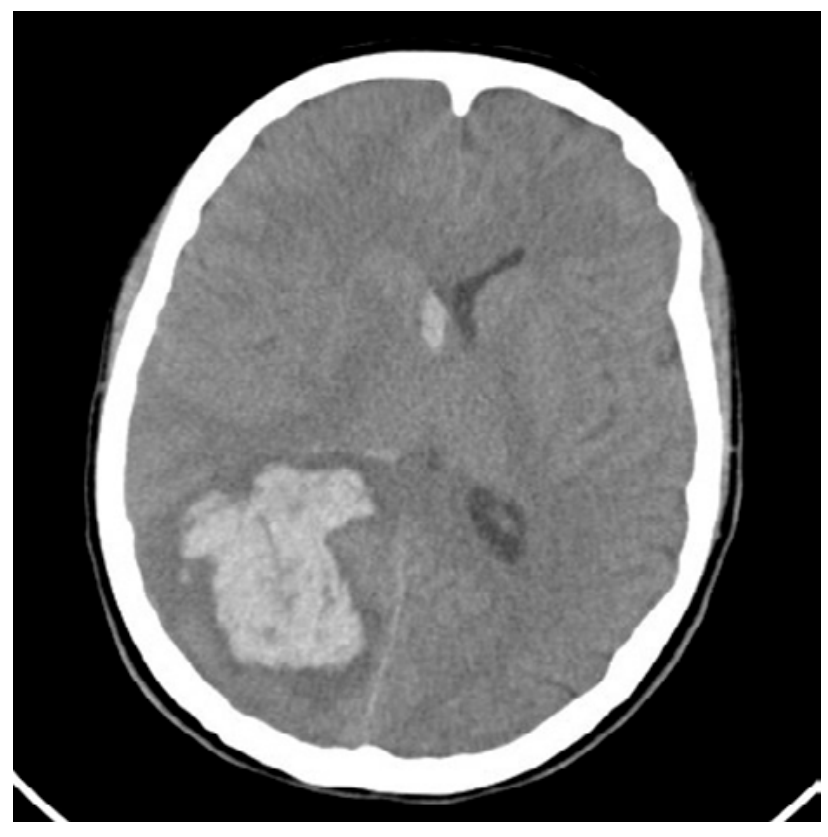

Figure 2. Angiography of the mycotic aneurysm in coronal and sagital plane

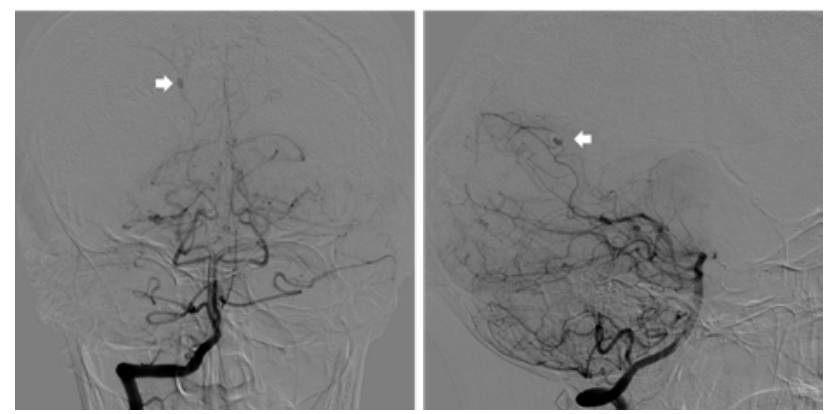

After 4 weeks under antibiotic therapy and with a good neurological evolution the patient was submitted to mitral valvuloplasty. No postoperative complication occurred. The histopathology of the excised material revealed fibrin and an extensive polymorphonuclear inflammatory infiltrate, the fungal research with periodic acid-schiff and Grocott's silver stain was negative.

The patient fulfilled a total of 6 weeks with the same empirical antibiotic therapy initially started without further neurological aggravation and improvement of left homonymous hemianopsia.

\section{DISCUSSION}

$M A$, despite the term, are in fact pseudo aneurysms since there is no involvement of all layers of the vascular wall. The most consensual theory for degeneration of the vascular wall results from septic embolization through the vasa vasorum severe inflammation of the adventitia, with intimal proliferation and destruction of internal elastic lamina?

As mentioned above, MA are a rare complication of $\mathrm{IE}$, with an incidence of 2 to $3 \%{ }^{3}$. The development of these aneurysmal formations is more frequently associated with bacterial subacute IE, predominating the Streptococcus viridans and Staphylococcus aureus as aetiological agents. Other agents, such as mycobacteria, viruses or fungi, may be also involved. The most frequently described symptoms are headache, fever, vomiting, ocular palsy, convulsions, hemiparesis, drowsiness or loss of consciousness ${ }^{3}$.

In this case, the patient presented a new mitral regurgitation, positive echocardiogram for $\mathbb{E}$, fever and vascular phenomena, totalling 1 major and 2 minor criteria of modified Duke criteria, allowing the probable IE diagnosis. The histopathology after valve surgery revealed fibrin tissue and an extensive polymorphonuclear inflammatory infiltrate, without microorganism identification, however, it is important to remind the patient was under 4 weeks of empirical antibiotic therapy for IE at that time. The negative extend blood cultures may be explained by the patient's antibiotic use at the hospital admission. The serologic and autoimmune study was negative, only revealing the patient a high Antistreptolysin 0 title that may raise the suspicion of a Streptococcus group bacteria involvement.

The ruptured aneurism with intraparenchymal haemorrhage at admission is frequently related to a high morbidity and mortality. An early neurosurgical intervention is vital for the favourable outcome. In recent cases, morbidity and mortality rates have been decreasing due to neurosurgical advances ${ }^{4}$.

The severe mitral regurgitation triggered by the IE also lacked surgical intervention, but the aneurysm rupture has, as well, implications at this approach. In the unruptured aneurysm, cardiac surgery can be performed without additional risk. The decision if cardiac or cerebral intervention should be performed first is based on the evaluation of aneurysm haemorrhagic risk versus heart failure severity. In the presence of a recent treated ruptured aneurysm, the haemorrhagic risk of valvuloplasty surgery is high, since it involves heparin-coated extracorporeal circulation, therefore, it is recommended to delay the cardiac intervention. In the literature, studies indicate a period superior to one month as ideal time delay. 5 This interval may differ depending on the magnitude of heart failure, so an individualized evaluation is imperative.

In conclusion, the authors emphasize the importance of the early diagnosis in IE and, although rare, ruptured cerebral MA are a challenging complication, demanding a patient centred approach.

\section{REFERENCES}

1. Osler W. The Gulstonian lectures on malignant endocarditis, Br Med J, 1885; vol.1:467-91.

2. Kannoth S and Thomas SV. Intracranial microbial aneurysm (infectious aneurysm): current options for diagnosis and management. Neurocritical Care,2009 vol.11(1):120-129.

3. Kuo I, Long T, Nguyen N, Chaudry B, Karp M, and Sanossian N. Ruptured Intracranial Mycotic Aneurysm in Infective Endocarditis: A Natural History. Case Reports in Medicine, 2010, ID168408.

4. Kannoth S, lyer R, Thomas SV, Furtado SV, Rajesh BJ, Kesavadas C et al. Intracranial infectious aneurysm: Presentation, management and outcome. J. Neurol. Sci, Vol:256 (1):3-9.

5. Rossi M, Gallo A, De Silva RJ, Sayeed R. What is the optimal timing for surgery in infective endocarditis with cerebrovascular complications? Interact Cardiovasc Thorac Surg. 2011;14(1):72-80. 